$$
666.94 .004 \cdot 1=60
$$

\title{
consideraciones acerca del pliego oficial español para la recepción de aglomerantes hidráulicos*
}

\author{
DOMINGO GUINEA GUERRERO \\ del Laboratorio de Ingenieros del Ejército.
}

Hemos aceptado la invitación, que agradecemos, del Instituto Técnico de la Construcción y del Cemento, para tener el honor de exponer ante ustedes algunas observaciones sobre los métodos de ensayo usados hoy en España para calificar el cemento portland. No intentamos con ellas hacer una revisión completa de los mismos, y menos ofrecer conclusiones precisas sobre cada uno de ellos, que pudieran servir de base para la confección de un nuevo Pliego. La misión del Laboratorio de Ingenieros del Ejército, es efectuar los ensayos de acuerdo con los métodos que señalan los que lo solicitan, no estudiar la razón y bondad de cada uno; es decir, aplicar la ley y no legislar. Pero como cn esta misión existe para los que la cumplen el grave peligro de adocenamiento y rutina, somos modestos continuadores de una tradición, transmitida a nosotros por don Félix González (q. e. p. d.), primer Director del Instituto del Cemento, y por don Palslo García de Paredes, primer Quimico de dicho Instituto, que consiste en dedicar el tiempo libre, cuando lo hay, a una critica constructiva de los métodos de ensayo empleados. Esta razón y nuestra postura, absolu-

(*) Ponencia presentada en los II Coloquios de Directores y Técnicos de Fúbricrs de Cemento. I. T. C. C., marzo $105 \pi$. tamente independiente $y$ equidistante de fabricantes y consumidores, les explicará nuestra presencia y actuación en este coloquio.

Sentaclo esto, paso a tratar de los ensayos a que se somete un cemento.

\section{Mortero normal y sus resistencias} MECÁNICAS

Admitido con carácter intcrnacional, el que las resistencias mecánicas del mortero normal de cemento sirvan para calificar, con este punto de vista, el cemento con clue está hecho, creemos de la mayor importancia todo cuanto se refiere a componentes, dosificación, amasado, tipo y confección de las probetas y rotura de las mismas.

Arena normal.-La calidad de la arena influye mucho en las resistencias del mortero. Un ejemplo claro de esto es que al sustituir la arena de Leucate por la del río Manzanares, hubo necesidad de aumentar la riqueza en cemento del mortero, para conservar los valores de sus resistencias mecánicas, pasando de una relación cemento :arena $=1: 3$, at la $1: 2,32$ que hoy se usa. 
La adopción de una arena normal comparable a las A. S. T. M., DIN y B. S., es problema fundamental y de solución no fácil. El Pliego Oficial tiene una definición ridícula de la arena normal, del río Manzanares, y esta arena, además de contener arcilla y mica, no es estable frente a los ácidos ni en el tiempo.

En algunos estudios hemos empleado arena de contenido en sílice superior al $98 \%$, moliendo grava - cuarcita- en un molino de bolas y en un molino de cilindros. Los granos obtenidos en el molino de bolas eran redondeados, y Mr. Bogue los consideró muy parecidos a los de la arena de Otawa, pero sus superficies, aunque presentaban la ventaja de encontrarse siempre en las mismas condiciones, nuevas y limpias, resultaban rugosas y los morteros poco plásticos. Además, y es lógico, un molino de bolas produce un gran tanto por ciento de finos, luego su rendimiento como fábrica de arena monograntlar o continua sin finos es malo. El molino de cilindro, de mejor rendimiento, da granos irregulares y de superficies aún más rugosas. Hemos intentado suavizar estas superficies por diferentes procedimientos, pero sin éxito.

Fracitsados en muestros intentos de lograr una arena normal artificial, consideramos cometido de geólogos el hallar una arena natural de la calidad de las extranjeras antes citadas, cuya especificación fundamental podría ser una pequeña velocidad inicial de disolución en ácido fluorhídrico. Creemos firmemente que, mientras no se adopte una lnena arena normal, no tiene sentido pensar en un nuevo Pliego.

Nos parecen aceptables las granulometrías de las arenas DIN y A. S. T. M. para compresión, así como ha propuesta por R. I. L.
E. M., si se incluye una fuerte limitación de su contenido de granos finos.

Agua de amasado.--En lá determinación del agua de amasado, cle la pasta pura, para lograr una plasticidad definida -agua de consistencia normal- se observa que, si se hace el amasado con paletín, los resultados dependen bastante del operador. Amasado a mano, con guantes de goma, los resultados son más concordantes y menor la fatiga del operador. Sin emlargo, parece preferihle usar una amasadora-batidora mecánica.

La sonda tiene sensibilidad suficiente para definir una cierta plasticidad en la pasta pura de cẹmento.

En cuanto al agua de amasado del mortero, preferimos calcularia mediante una función lineal, bien estudiada, del agua de consistencia normal, a determinarla con una mesa de sacudidas, fijada la plasticidad de la masa ; el ensayo con la mesa de sacudidas no tiene gran sensibilidad.

Dosificación.-De acuerio con que la relación cemento/arena sea $1: 3$ y no $1: 2,32$, creemos indispensable, que la masa del mortero tenga consistencia plástica, para que las resistencias mecánicas del mortero normal sean comparables, o mejor análogas, a las de un buen hormigón hecho con el mismo cemento. Es cierto, que las resistencias de nuestros cementos parecerían haberse hundido a1 cambiar de Pliego, pero más cierto es que jamás un hormigón puede alcanzar las resistencias que hoy figuran en los informes oficiales, datos éstos quie en algunos constructores pueden producir confusiones peligrosas. Además, a los alemanes se les presentó idéntico problema al adoptar la Norma DIN 1164 , y lo resolvieron solapando la vi-. 
gencia de los dos Pliegos, antigno y nuevo, durante algún tiempo.

Anasado.-La amasadora propuestá por R. I. L. E. M. no es satisfactoria. Su amasado y mezclado es poco enérgico e imperfecto. La amasadora DIN, la que hoy se usa en España, tiene el peligro de permitir una gran evaporación del agua de itmátolo si el ambiente es seco.

No creemos dificil que cualquiera de las dos, con ligens modificaciones, pudiera ser aceptada.

Probetas'de ensayo. Confección y rotura. Nos inclinamos hacia la probeta DIN, prismática, de $4 \times 4 \times 10 \mathrm{~cm}^{3}$. Las probetas A. S. T. M., con forma de ocho, rompen mal, y las antiguas DIN, de forma parecida : las del Pliego Español, rompen mejor gracias a su fuerte estrangulamiento, pero este estrangulamiento, precisamente, crea una distribución de tensiones tal en la zona de rotura, que el vaior hallado tiene interpretación difícil y es muy inferior a la verdadera resistencia a la tracción. Con las probetas prismáticas, en cambio, se obtienen valores más concordantes y reales, tanto en tracción por fiexión como en la rotura por aplastamiento -compresión- de cada tino de los trozos que resultan del primer ensayo.

Es muy impartante fijar en estos ensavos la velocidad de carga, pues influye mucho en los resultados. También es necesario señalar la dureza de las platinas de compresión: nosotros usamos platinas muy duras cromadas con cromo duro.

Acerca de la confección de las probetas, las mejores concordancias las hemos logra- do apisonando a mano, según DIN 1164. La apisonadora mecánica R. I. I. E. M. no nos satisface, quizá por no haber empleado masas, cuya plasticidad convenga a la máquina.

\section{ENSAYOS FISICOQUĹMICOS}

Tiempo de fraguado.-Si bien las determinaciones de la aguja de Vicat carecen de sentido físico, el procedimiento es bueno, sobre todo si se usa un sistema automático registrador, para medir los tiempos de endurecimiento y fraguado, cosas que no necesitan gran precisión.

Superficie especifica $y$ finura de molido.Es uno de los temas más estudiados por nosotros. Antes de estar normalizados los aparatos de Lea (B. S.) y de Blaine (A. S. T. M.), ya los utilizábamos tratando de encontrar una sustitución de los actuales ensayos de tamizado en seco con los tamices de 900 y 4.900 mallas $/ \mathrm{cm}^{2}$. Las partículas activas del cemento tienen, cuando más, un diámetro de unas treinta micras, y la menor lu $z$ de nuestros tamices es 89 micras. Dos cementos ptieden dar el misno residuo cn $4.900 \mathrm{mallas} / \mathrm{cm}^{2}, y$ poseer, sin embargo, proporciones completamente diferentes de las citadas partículas, es decir, de aglomerante efectivo. Si a esto agregamos el que los residuos son del orden del $10 \%, \operatorname{los}$ tamices mal pueden calificar al 90 por 100 restante, y debe, pues, desecharse el procedimiento.

Los medidores de superficie especifica Blaine y Lea, basados en la determinación de la resistencia que una pastilla de polvo de cemento opone al paso de una corriente de aire, son interesantes, sencillos, rápidos y de resultados muy concordantes. Un aparato 
necesita muestra patrón y otro no y, á pesar de esta diferencia, reproducen las medidas. Los creemos ideales, sobre todo el Blaine, para la vigilancia de fábrica, si bien no los consideramos adeccuados para señalar con ellos una especificación del cemento. El reparo grave que oponemos es el siguiente:

En uil cuerpo pulverulento monogranular, la superficie es inversamente proporcional a la dimensión transversal elegida en el grano, lo cual quiere decir que la superficie específica aumenta muy deprisa al disminuir el tamaño del grano. Por tanto, el numero que resulta, si se emplea uno de estos aparatos, depende, de modo casi exclusivo, de la parte más finamente molida del cemento. Tos cementos españoles son, desgraciadamente, ricos en aluminato tricálcico, aproximadamente entre el 7 por 100 y el 20 por 100 , y los efectos perjudiciales de este componente se pueden contrarrestar en parte, según diremos más adelante, mediante una adición conveniente de yeso, cantictad que varía mucho de un cemento a otro. Este yeso se ha molido con el clínquer y es mucho más b'ando que él, luego estará mucho más molico. La superficie específica medida está estrechamente ligada a la proporción de finos, o sea, a la de yeso.

Como cjentlo polemos citar: Que nos ha sido mucho más fácil alcanzar una superficie específica Blaine de $8.000 \mathrm{~cm}^{2} / g$ en $u$ cemento de 9 por 100 de yeso, que una sitperficie especifica de $4.000 \mathrm{~cm}^{2} / \mathrm{g}$ en otro con 4 por 100 de yeso. Además, aunque dos cementos tuvieran la misma proporción de yeso, por ejemplo, nula, la stiperficie especifica dependería casi únicamente de la prom porción de granos finísimos, cuya velocidad de reacción es tan grande, que constituyen la fracción desagradable, por explosiva, del cemento.
Resumiendo, los tamices se quedan cortos y los permeabilímetros largos: a través de los primeros pasa la fracción interesante $y$ algo más; en los segundos, la medida está fuertemente influtda por la fracción más fina, que no interesa como aglomerante.

Los aparatos que estudian una sedimentación son: tubos de Wiegner, método engorroso y poco preciso; pipeta de Andreasen, con idétnticas características; el mal llamado fluorómetro, muy lento; y los turbidínetros, buenos pero caros, lentos y delicados, sólo apropiados para investigación y controversia.

Nuestra propuesta cs la siguiente: Como la curva de frecuencias de tamaño de partículas, que caracteriza a uno cualquiera de los molinos que se emplean, es una recta en el diagrama de Rosin-Ramler, đos ensayos de tamizado con tamices de $7 t$ micras y $44 \mathrm{mi}$ cras, o mejor con 74 micras y 37 micras, definirian dicha recta en el citado diagrama $y$ permitirían calcular la superficie total de las partículas, supuestas esféricas como en los turbidímetros, contenidas en un gramo de muestra, y cuyos diámetros estuvieran comprendidos entre 1 micra y 30 micras, o entre otros dos limites lógicamente aceptables. Así mediriamos la stperficie especifica de la fracción ítil del cemento. Los tamizados se deben realizar en corriente de agua; son rápidos y concordantes.

Estabilidad de volumen--Este toma es delicado, por los fuertes comentarios oidos y lé́dos acerca del método A. S. T. M., del curado ell autoclave. El mútodo es lógico, pues consiste, como saben, en un curado acelerado a $215^{\circ} \mathrm{C}$. Y que no es tan feroz como algunos piensan, lo demuestra el que, en la seric de cementos enviada el año pa- 
sado al Laboratorio de Ingenieros del Ejército por la Delegación del Gobierno en la Industria del Cemento, de 43 cementos, cumplen 26 la condición A. S. T. M., o sea, el 60 por 100. Quizá se pudiera pensar en efectuar el curado a una temperatura algo inferior, o, mejor, en aumentar la expansión tolerable en los cementos españoles. Lo cierto es que los métodos que hoy usamos, tortas o galletas y cilindros, carecen de sensibilidad, y no conocemos, aparte del DIN, ningún otro. Para corroborar lo último, diremos, que un cemento que presenta buena estabilidad, según el Pliego Español, tuto en autoclave una expansión del 11 por 100 .

El yeso en el cemento.-Parece demostrado, y nosotros lo hemos confirmado con ensayos de retracción DIN, y medida de resistencias mecánicas, que un buen ajuste de la cantidad de yeso añadido al clínquer, mejora la calidad del cemento obtenido. In misión del yeso en el clínquer consiste en actuar de retardador, y, además, netutralizar parte de los efectos perjudiciales del aluminato tricálcico. Dosificar el yeso con la aguja de Vicat equivale a desconocer su segundo papel, tan importante como el primero en cementos que presentan un contenido medio de $C_{3} A$ del 13 por 100 , valor que se observa tiende a disminuir, gracias, probablemente, a 171 esfuerzo constante de los fabricantes.

De acuerdo con lo anterior; nos gustaría que desapareciera la cota stoperior de contenido en $\mathrm{SO}_{3}, y$ se sustituyera por el ensayo de Lereh.

Calor de fraguado.-Su determinación sólo interesa si el cemento se va a emplear para fabricar grandes masas de hormigón: presas y fortificaciones. EI método A. S. T. M. es satisfactorio.

\section{II. ANátisis QUfMICo}

Composición del cemento.--El análisis quimico necesario pára conocer su composición elemental, valorada en óxidos, parece logrado. Dichos números anticipan poco acerca de las propiedades del cemento. Tampoco parecen servir para gran cosa los indices hidráulico y alumínico. A pesar de todos los reparos, la información más completa la dan hoy las fórmulas de Bogue, que permiten calcular la composición potencial. Es cierto que están pensadas para un sistema en equilibrio, suponiendo, por tanto, la ausencia de fases metastables, pero entre lo normalizado parece lo mejor, y no tenemos grandes esperanzas de que puedan ser sustituidas a corto plazo por alguna otra cosa.

Alcalis y cal libre.-Son dos determinaciones importantes que no están incluidas en nuestro Pliego. Si estimamos como cierto que los álcalis influyen desfavorablemente en la durabilidad del mortero u hormigón, y que la cal libre es la principal causante de la expansión, debemos prestar mucha atención a estos dos ensayos. También sirven para indicar el grado de cocción del clinquer.

Deben estudiarse estos problemas para completar la especificación del cemento portland.

Pérdida al fuego y residuo insoluble.-Son indices de cocción del clinquer y conservación del cemento, pero de mucha menos jm. portancia que los anteriores. Pueden conservarse por tridición.

\section{RESU M N}

Hemos expuesto todos los puntos en los qute, a nuestro leal saber y entender, se debe. 
pensar antes de atreverse a redactar in nuevo Pliego. No damos a todos el mismo peso; nos parecen principales y urgentes los que se refieren a una arena tipo, ensayos con el mortero normal, tiempo de fraguado del cemento, yeso en el cemento y estabilidad de volumen o expansión. Otros son complementarios: finura de molido, calor de fraguado y composición del cemento. $Y$ de los relativos a contenido en álcalis, y cal libre, sospechamos sólo su importancia, pero aún no tenemos datos stificientes.

El Laboratorio de Ingenieros del Ejercito, que dirige el Coronel Ingeniero de Construc- ciún, don Ricardo liscudero Cisneros, ticne una larga experiencia en todo lo relacionado con los ensayos de cementos. Se han hecho y se siguen haciendo bastantes trabajos de investigación relacionados con los mismos, a cargo de un equipo formado por el Comandante Jiménez Montoya, señor Guinea y un servidor. Estamos a disposición de todos los fabricantes de cementos, así como de los Organismos Oficiales, para todo cuanto pueda contribuir a la mejora del Pliego, que tanta falta está haciendo a la indisstria, ya que son necesarias especificaciones más reales $\mathrm{y}$, a veces, con métodos más sencillos gute los actuales. 